\title{
ARTí́cUlo \\ Variaciones temporales de las defensas antioxidantes e índices proteína:ADN y ARN:ADN en el poliqueto Eurythoe complanata
}

Temporal variations of antioxidant defenses and protein:DNA and RNA:DNA indices in the polychaete Eurythoe complanata

\author{
Edgar Zapata-Vívenes ${ }^{1 *}$, Osmar Nusetti ${ }^{1}$ y Leida Marcano ${ }^{1}$
}

\begin{abstract}
'Departamento de Biología, Escuela de Ciencias, Núcleo de Sucre, Universidad de Oriente, Avenida Universidad, Cerro Colorado Cumaná, Venezuela. *ezapatavivenes@gmail.com

Abstract.- Some antioxidant responses and molecular index of growth (protein:DNA and RNA:DNA) were determined in the polychaete Eurythoe complanata collected from Gulf of Cariaco (Venezuela) during December $2009\left(26.03 \pm 0.64^{\circ} \mathrm{C}\right)$, February $\left(23.96 \pm 0.67^{\circ} \mathrm{C}\right)$, June $\left(27.82 \pm 0.50^{\circ} \mathrm{C}\right)$ and October $2010\left(29.93 \pm 0.37^{\circ} \mathrm{C}\right)$. Also, the number of individuals with signs of tissue regeneration was counted. Concomitantly, a bioassay of acclimatization was carried out at 24 and $28^{\circ} \mathrm{C}$ during 36 days. Muscular tissue was used to determine enzymatic activities of catalase (CAT), glucose-6-P dehydrogenase (G6PDH), glutathione-s-transferase (GST); oxidative damage (TBARS), metallothionein (MT) and thiols (-SH) concentrations. DNA, RNA and proteins (PT) concentrations and their related indexes were determined. During 'no upwelling' season (June and October 2010), when the mean temperature exceeds $28^{\circ} \mathrm{C}$, there were increases in CAT, TBARS and DNA. G6PDH, GST, MT and -SH did not vary seasonally or in acclimatizing bioassays. Similarly, in this season a number of individuals collected with new setigers were observed. RNA:DNA and PT:DNA indices demonstrate a continuous growth of the species throughout the year and a significant increase of protein synthesis in low temperatures season (upwelling), respectively. Somatic growth, lipoperoxidative damage and antioxidant capacity in E. complanata are stimulated in seasons of maximal temperature.
\end{abstract}

Key word: Molecular indices, temperature, catalase, lipid peroxidation

\begin{abstract}
Resumen.- Algunas respuestas antioxidantes e índices instantáneos de condición (proteínas:ADN y ARN:ADN) fueron determinados en el poliqueto Eurythoe complanata colectado en el Golfo de Cariaco (Venezuela) durante diciembre 2009 $\left(26,03 \pm 0,64^{\circ} \mathrm{C}\right)$; febrero $\left(23,96 \pm 0,67^{\circ} \mathrm{C}\right)$, junio $\left(27,82 \pm 0,50^{\circ} \mathrm{C}\right)$ y octubre $2010\left(29,93 \pm 0,37^{\circ} \mathrm{C}\right)$. También se estimó el número de ejemplares colectados con señales de regeneración tisular. Paralelamente, fue llevado a cabo un bioensayo de aclimatación a 24 y $28^{\circ} \mathrm{C}$ por un lapso de 36 días. El tejido muscular se usó para determinar las actividades de las enzimas: catalasa (CAT), glucosa-6-P deshidrogenasa (G6PDH), glutatión-S-transferasa (GST); y niveles de daño oxidativo (TBARS), metalotioneínas (MT) y grupos tioles totales (-SH). Además, fueron determinados los niveles de ADN, ARN y proteínas, y sus índices relacionados. En la temporada de no surgencia (junio y octubre 2010), caracterizada por temperaturas promedio que sobrepasan los $28^{\circ} \mathrm{C}$, fueron observados incrementos en CAT, TBARS y ADN. Sin embargo, las actividades de G6PDH, GST, MT y -SH no presentaron diferencias estacionales ni variaron en los bioensayos de aclimatación. De igual forma, en la temporada de no surgencia se observó un mayor número de individuos con señales de regeneración. Los índices ARN:ADN y PT:ADN demuestran un crecimiento continuo de la especie durante todo el año y un aumento en la síntesis de proteínas sólo en temporadas de bajas temperaturas (surgencia), respectivamente. La capacidad antioxidante, el daño lipoperoxidativo y el crecimiento somático en E. complanata son estimulados en las temporadas de temperaturas máximas.
\end{abstract}

Palabras clave: Índices moleculares, temperatura, catalasa, peroxidación lipídica

\section{INTRODUCCIÓN}

Eurythoe complanata (Pallas, 1766) es un poliqueto errante de relativa abundancia en el golfo de Cariaco (Venezuela), que habita asociado a arrecifes coralinos, fondos arenosos someros, debajo de piedras y corales muertos (Liñero-Arana \& Díaz 2010). E. complanata es una especie que ha sido utilizada como modelo biológico en la estandarización de protocolos ecotoxicológicos, a través de biomarcadores moleculares y celulares (sistema inmune y antioxidante, poder de regeneración de tejidos, y la capacidad corporal de acumular xenobióticos) aplicados a la evaluación de sedimentos marino-costeros impactados por contaminantes (Méndez \& Páez-Osuna 1998, Nusetti et al. 1998, 2005; Zapata-Vívenes et al. 2005, Vásquez-Núñez et al. 2007). Sin embargo, no existe información sobre sus respuestas bioquímicas basales mediadas por los cambios ambientales, en especialmente la temperatura. 
Las temperaturas en el golfo de Cariaco varían asociadas a eventos de surgencia costera, oscilando entre 20 a $24^{\circ} \mathrm{C}$ durante enero a junio (surgencia), para luego observarse un ascenso de temperatura de 27 a $29^{\circ} \mathrm{C}$ a partir de julio y hasta principios de noviembre (no surgencia) (Penchaszadeh et al. 2000, Rueda-Roa \& Muller-Karger 2013). Se conoce que las fluctuaciones térmicas que se registran en el agua de mar inciden directamente en la temperatura corporal de $E$. complanata (Kudenov 1974), siendo éste un factor ambiental influyente en la actividad basal y tasa metabólica de los organismos marinos (Guderley \& St-Pierre 2002, Keller et al. 2004).

La temperatura del mar influye directamente el control respiratorio y crecimiento en los organismos; tales procesos fisiológicos pueden conllevar la formación de especies reactivas del oxígeno (ERO) (Abele \& Puntarulo 2004, Buttemer et al. 2010, Freire et al. 2011). La sobreproducción de ERO puede causar daños a nivel molecular tales como peroxidación de los lípidos de membranas, daños al ADN e inhibición enzimática (Hermes-Lima 2004). Sin embargo, existen una gama de moléculas antioxidantes que pueden atenuar la acción de las ERO, por ejemplo compuestos ricos en grupos sulfhidrilos (glutatión reducido-SH y metalotioneínas-MT) y las enzimas superóxido dismutasa (SOD), catalasa (CAT), glutatión reductasa (GR) y glutatión peroxidasa (GPx). Adicionalmente, se encuentra la enzima glutatión-S-transferasa (GST) que utiliza como sustrato al glutatión reducido (GSH) para facilitar la ruta de desintoxicación de xenobióticos y de compuestos endógenos como lípidos peroxidados (Oakley 2011). También, la enzima glucosa-6-P deshidrogenasa (G6PDH) produce NADPH y pentosas que funcionan como poder reductor para la enzima glutatión reductasa (GR) y para la síntesis de ácidos nucleícos, respectivamente (Singh et al. 2012).

El crecimiento de los organismos es una medida integral de su metabolismo, siendo sensible a factores estresantes externos. E. complanata es un organismo de crecimiento corporal continuo con un alto poder de regeneración (ZapataVívenes 2001). Este proceso implica una inversión de moléculas energéticas vinculadas con la síntesis de biomoléculas destinadas a la división y crecimiento celular, lo que conlleva a cambios en su composición bioquímica (YáñezRivera \& Méndez 2014). De igual manera, la determinación de los niveles de macromoléculas relacionadas (proteínas, ARN y ADN) y sus respectivos cocientes se ha hecho de uso extensivo como índices moleculares de condición en organismos acuáticos (Nusetti \& Morales 1988). Estos cocientes biomoleculares pueden ser afacetados por la condición nutricional del organismo (Mendoza et al. 2002), cambios de la temperatura (Lemus \& Chung 2006) y presencia de tóxicos (Zapata-Vívenes et al. 2015).

Se hace necesario conocer cómo pueden ser influidas las respuestas antioxidantes e índices instantáneos de condición por los cambios temporales de la temperatura, tales parámetros moleculares son comúnmente referidos como biomarcadores o herramientas para la evaluación de calidad ambiental. En referencia a lo antes expuesto, en esta investigación se evaluó como varían estacionalmente las respuestas antioxidantes, daño oxidativo a lípidos e índices moleculares de crecimiento en el poliqueto $E$. complanata colectados en distintas temporadas ambientales.

\section{MATERIALES Y MÉTODOS}

\section{Organismos}

Los ejemplares de Eurythoe complanata fueron colectados en aguas someras de la franja costera de la zona sur del Golfo de Cariaco, en las adyacencias de la ensenada de Turpialito (11 $\left.{ }^{\circ} 22^{\prime} 30^{\prime \prime} \mathrm{N}, 64^{\circ} 04^{\prime} 50^{\prime \prime} \mathrm{O}\right)$, durante diciembre 2009 (26,03 $\left.\pm 0,64^{\circ} \mathrm{C}\right)$, febrero $\left(23,96 \pm 0,67^{\circ} \mathrm{C}\right)$, junio $\left(27,82 \pm 0,50^{\circ} \mathrm{C}\right)$ y octubre $2010\left(29,93 \pm 0,37^{\circ} \mathrm{C}\right)$ (Fig. 1). Al mismo tiempo, se estimó el porcentaje individuos colectados con signos de regeneración y número de nuevos setígeros como índices de crecimiento.

Una vez trasportados al laboratorio, a cada individuo se le disecó el tracto intestinal, y su porción muscular (tegumento) fue almacenada durante 3 días a $-20^{\circ} \mathrm{C}$. Durante los meses de colectas se usó un termógrafo para estimar la temperatura del agua de mar.

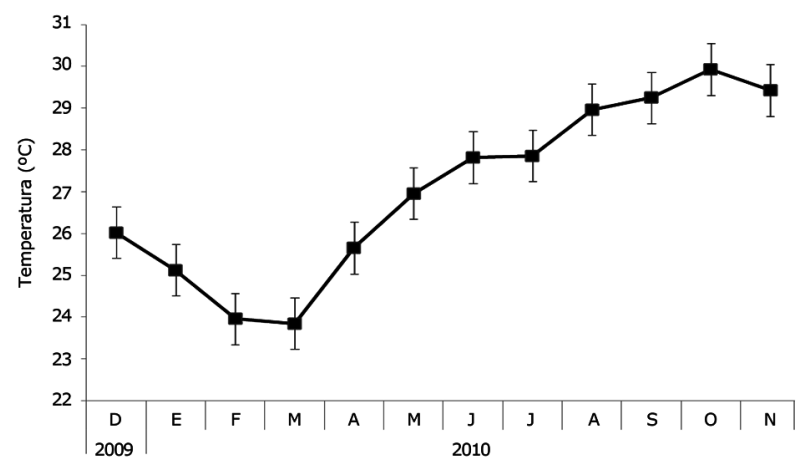

Figura 1. Registro de temperaturas durante diciembre 2009 a noviembre 2010 en el Golfo de Cariaco / Temperatures during December 2009 to November 2010 in the Gulf of Cariaco 


\section{Bioensayo REFERENCIAL}

Dos grupos de organismos $(n=20$; por triplicado) fueron mantenidos, por separados, en acuarios a diferentes temperaturas $24^{\circ} \mathrm{C}$ y $28^{\circ} \mathrm{C}$ (variación diaria entre 0,1 a $0,6^{\circ} \mathrm{C}$ ) por un período de 36 días. Tales temperaturas simulan los promedios en las épocas de surgencia y estratificación (no surgencia) que ocurren en el Golfo de Cariaco, respectivamente. Tanto en los organismos colectados en las distintas temporadas como en los ejemplares aclimatados a las dos temperaturas se procedió a medir los niveles de compuestos antioxidantes (metalotioneínas -MT y grupos sulfhidrilos totales -SH), daño oxidativo a lípidos, actividad enzimática de G6PDH, GST y CAT; ADN, ARN y proteínas, y sus respectivos cocientes relacionados. También fue estimado el porcentaje individuos con signos de regeneración

\section{Metalotioneínas (MT)}

Los tejidos se homogenizaron en proporción 1:8 en buffer Tris$\mathrm{HCl} 20 \mathrm{~mol} \mathrm{~L}^{-1} \mathrm{pH} 8,6$, contentivo de sacarosa $500 \mathrm{mmol} \mathrm{L}^{-1}$,

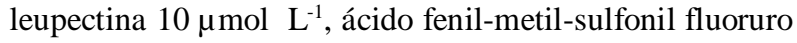
(PMSF) $0,5 \mathrm{mmol} \mathrm{L}^{-1} \mathrm{y}$ ditiotreitol (DTT) $2 \mathrm{mmol} \mathrm{L}^{-1} \mathrm{a} 4^{\circ} \mathrm{C}$. El homogenizado fue centrifugado a $2.000 \mathrm{~g}$ por $30 \mathrm{~min}$. Por cada mililitro del sobrenadante se le adicionó $1,5 \mathrm{~mL}$ de etanol absoluto $\left(-20^{\circ} \mathrm{C}\right)$ y $80 \mu \mathrm{L}$ de cloroformo, y luego se centrifugó a $6.000 \mathrm{~g}$ por $10 \mathrm{~min}\left(4^{\circ} \mathrm{C}\right)$. El sobrenadante resultante se mezcló con $40 \mu \mathrm{L}$ de $\mathrm{HCl}$ al $37 \%$ y $3 \mathrm{~mL}$ de etanol frío, y fue mantenido a $-20^{\circ} \mathrm{C}$ por 1 hora. Una nueva centrifugación se realizó a $6.000 \mathrm{~g}$ por $10 \mathrm{~min}$. Posteriormente, el precipitado obtenido se resuspendió con etanol $87 \%$, cloroformo $1 \%$ y buffer de homogenización, y posteriormente se centrifugó a $6.000 \mathrm{~g}$ por $10 \mathrm{~min}$. El precipitado se mezcló con $150 \mu \mathrm{L}$ de

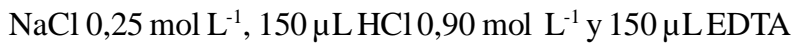

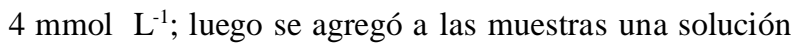

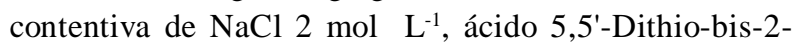
nitrobenzoico (DTNB) a $0,4 \mathrm{mmol} \mathrm{L}^{-1}$ y buffer fosfato de sodio $200 \mathrm{mmol} \mathrm{L}^{-1} \mathrm{pH} 8,6$. La mezcla de reacción se incubó a temperatura ambiente y protegidas de la luz durante $30 \mathrm{~min}$, luego se centrifugó a $3.000 \mathrm{~g}$ x $30 \mathrm{~min}$. Se estimaron los niveles de MT ( $\mu$ moles de MT g ${ }^{-1}$ de masa húmeda) utilizando una curva patrón de glutatión reducido $(\mathrm{GSH})$ a $412 \mathrm{~nm}$ (Viarengo et al. 1997).

\section{Tioles totales (-SH)}

La determinación de tioles solubles se realizó por método de Ellman (1958). Se tomaron $100 \mathrm{mg}$ de las distintas regiones corporales, se homogenizaron con $2 \mathrm{~mL}$ del buffer $100 \mathrm{mmol}$ $\mathrm{L}^{-1}$ Tris ácido etilendiamino-tetracetato (EDTA) $\mathrm{pH}$ 8,0. Se centrifugó a $5.000 \mathrm{~g}$ durante $5 \mathrm{~min}$. Del sobrenadante obtenido, se tomaron $200 \mu \mathrm{L}$ más $200 \mu \mathrm{L}$ de ácido tricloroacético al $10 \%$ (ATC) y se colocó a $4^{\circ} \mathrm{C}$ por $15 \mathrm{~min}$, luego se centrifugó durante $15 \mathrm{~min}$ a $2.000 \mathrm{~g}$. Se tomaron $200 \mu \mathrm{L}$ del sobrenadante y se le agregaron $800 \mu \mathrm{L}$ de Tris- $\mathrm{HCl}$ pH 8,9 y $80 \mu \mathrm{L}$ de DTNB. Posteriormente, fueron leídos en un espectrofotómetro UV-VIS a $412 \mathrm{~nm}$, usando GSH como sustancia de referencia.

\section{Peroxidación de LíPIDOS}

La peroxidación lipídica se estimó por el protocolo propuesto por Ohkawa et al. (1979). Los tejidos fueron homogenizados en una concentración de $50 \mathrm{mg} \mathrm{mL}^{-1}$ en buffer fosfato de sodio

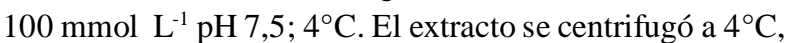
$6.000 \mathrm{~g}$ por $20 \mathrm{~min}$. Doscientos $\mu \mathrm{L}$ del homogeneizado se incubaron en baño de agua a $37^{\circ} \mathrm{C}$ con agitación constante durante $15 \mathrm{~min}$. A cada tubo se le añadió $250 \mu \mathrm{L}$ de una solución constituida por ATC $12,5 \%$ y $\mathrm{HCl} 0,8 \mathrm{~mol} \mathrm{~L}^{-1}$. Luego, se le agregó $500 \mu \mathrm{L}$ de solución de ácido tiobarbitúrico al $1 \%$. Se colocaron en un baño de agua a $90^{\circ} \mathrm{C}$ con agitación constante por $30 \mathrm{~min}$, y posteriormente colocados en baño de hielo por 10 min. Luego, se centrifugó a $1.500 \mathrm{~g}$ durante $10 \mathrm{~min}$ a temperatura ambiente. Las sobrenadantes de las muestras fueron leídas a $535 \mathrm{~nm}$. La concentración de las sustancias que reaccionan al ácido tiobarbitúrico (TBARS) se calculó usando 1,1,3,3-tetraetoxipropano como estándar.

\section{AnÁlisis enZimáticos}

Para la determinación de las actividades enzimáticas se usó el protocolo propuesto por Nusetti et al. (2001). Los tejidos se homogenizados en una relación 1:8 $\mathrm{m} \mathrm{v}^{-1}$ del buffer de Tris$\mathrm{HCl}$ (hidroxymetil-metilamina) $20 \mathrm{mmol} \mathrm{L}^{-1} \mathrm{pH}$ 7,6 conteniendo EDTA $1 \mathrm{mmol} \mathrm{L}^{-1}$, DTT $4 \mathrm{mmol} \mathrm{L}^{-1}$, sacarosa $500 \mathrm{mmol} \mathrm{L}^{-1}$, $\mathrm{KCl} 150 \mathrm{mmol} \mathrm{L}^{-1}$ y PMSF $1 \mathrm{mmol} \mathrm{L}{ }^{-1}$. El extracto resultante se centrifugó inicialmente a $2.000 \mathrm{~g}$ por $10 \mathrm{~min}$ a $4^{\circ} \mathrm{C}$. El sobrenadante se centrifugó nuevamente a $10.000 \mathrm{~g}$ durante 30 min a $4^{\circ} \mathrm{C}$. El volumen final colectado se usó como fuente de enzimas. La actividad de las enzimas antioxidantes se determinó en un espectrofotómetro Perkin Elmer UV/VIS Lambda 2S, bajo condiciones de temperatura controlada a $26 \pm 0,5^{\circ} \mathrm{C}$. Los ensayos enzimáticos fueron realizados por triplicado en un volumen final de $1,0 \mathrm{~mL}$.

Las estimaciones de pendiente y actividad enzimática específica ( $\mathrm{U} \mathrm{g}^{-1}$ de masa húmeda del tejido) se realizaron en el programa UV Winlab Lambda 25 bajo ambiente Windows. 
Glucosa-6-Fosfato Deshidrogenasa (G6PdH; EC 1.1.1149)

La velocidad de reacción se determinó midiendo el incremento en la absorbancia a $340 \mathrm{~nm}$ como resultado de la reducción de $\mathrm{NADP}^{+}$por min a $25^{\circ} \mathrm{C}$ a $\mathrm{pH} 7,8$. Para tal fin se añadió en la

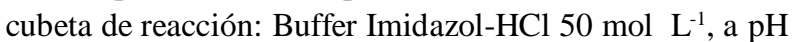
$7,5, \mathrm{MgSO}_{4} 5 \mathrm{~mol} \mathrm{~L}^{-1} ; 0,05 \mathrm{~mol} \mathrm{~L}^{-1} \mathrm{NADP}^{+}$y $100 \mu \mathrm{L}$ de extracto. La reacción se inició con glucosa-6-fosfato $5 \mathrm{mmol}$ $\mathrm{L}^{-1}$.

\section{GLUTATIÓN-S-TRANSFERASA (GST, EC 2.5.1.18)}

Se determinó siguiendo el ascenso en la concentración del complejo formado por GSH y 1-cloro-2,4-dinitrobenceno (CDNB), a una longitud de onda de $340 \mathrm{~nm}$ (Coef. de Extinción $\left.=9,6 \mathrm{mM}^{-1} \mathrm{~cm}^{-1}\right)$. Mezcla de incubación consistió en $\mathrm{Na}_{2} \mathrm{HPO}_{4} 100 \mathrm{mmol} \mathrm{L}^{-1} \mathrm{pH} 6,5$, CDNB 2,5 mmol L-1 disuelto en etanol, GSH $67 \mathrm{mmol} \mathrm{L}^{-1}$.

\section{Catalasa (CAT, EC.1.11.1.6)}

Se determinó por el descenso de la concentración del peróxido de hidrógeno $\left(\mathrm{H}_{2} \mathrm{O}_{2}\right)$ a una longitud de onda de $240 \mathrm{~nm}$ (Coef. de Extinción $\left.=40 \mathrm{M}^{-1} \mathrm{~cm}^{-1}\right)$. La mezcla de incubación estuvo constituida por buffer fosfato $\mathrm{KH}_{2} \mathrm{PO}_{4} / \mathrm{K}_{2} \mathrm{PO}_{4} 50 \mathrm{mmol} \mathrm{L}-1$ a $\mathrm{pH} \mathrm{7,5} \mathrm{y} \mathrm{H}_{2} \mathrm{O}_{2} 50 \mathrm{mmol} \mathrm{L}^{-1}$.

\section{BiOMOLÉCULAS E ÍNDICES MOLECULARES}

La concentración de proteínas (PT) fue valorada según el método de Lowry et al. (1951) usando albumina de suero de bovino como estándar. El ARN fue determinado por el método de revelación por Orcinol (Cambell \& Sargent 1967), yel ADN mediante la reacción de Difenilamina (Burton 1956); ambos valorizados en fosforo inorgánico (Buckley \& Bulow 1987). Se usó como sustancia referencial ARN de levadura $(S$. cerevicea) y ADN de timo de bovino. Adicionalmente, se estimaron los cocientes de crecimiento instantáneo ARN:ADN y Proteínas:ADN (Bulow 1987).

\section{Análisis estadísticos}

Después de análisis previos de normalidad de los datos y homogeneidad de las varianzas por métodos de Shapiro-Wilk y Levene (Sokal \& Rohlf 2012), respectivamente, se procedió a realizar un análisis de varianza de una vía (ANOVA) usando el programa computarizado Statgraphics Plus versión 5.1, en el cual se determinaron las diferencias entre las respuestas bioquímicas en los distintos meses de colectas y su contrastes con los bioensayos de aclimatación. Sólo las actividades de G6PDH, PT/ARN y ADN fueron ajustadas a Log10 para normalización de datos.
Tabla 1. Porcentaje de individuos con indicios de regeneración y número de segmentos (anteriores y/ o posteriores) en E. complanata colectados en los distintos meses. El asterisco $\left(^{*}\right)$ muestra diferencias significativas al $\mathbf{9 5 \%}$ de confianza / Percentage of individuals with regeneration indications and number of segments (anterior and posterior) in E. complanata collected in several months. The asterisk $\left({ }^{*}\right)$ shows significant differences to $95 \%$

\begin{tabular}{ccc}
\hline & $\begin{array}{c}\text { Porcentaje de } \\
\text { regeneración (\%) }\end{array}$ & $\begin{array}{c}\mathrm{N}^{\circ} \text { de } \\
\text { segmentos }\end{array}$ \\
\hline Diciembre & 32 & $6,90 \pm 2,07$ \\
Febrero & 33 & $6,63 \pm 1,92$ \\
Junio & 56 & $9,00 \pm 3,68 *$ \\
Octubre & 52 & $10,00 \pm 3,55 *$ \\
\hline
\end{tabular}

\section{RESUltados}

\section{Crecimiento}

El porcentaje en individuos colectados con indicios de regeneración (nuevos setígeros) en los distintos meses se presenta en la Tabla 1. Se observó que la regeneración es un proceso común en los individuos colectados en cualquier época del año, sin embargo los mayores promedios de número de setígeros recién formados por cada individuo $\left(\right.$ set ind $\left.^{-1}\right)$ fueron encontrados en junio $\left(56 \% ; 9,00 \pm 3,68\right.$ set ind $\left.^{-1}\right)$ y octubre $2009\left(52 \% ; 10,00 \pm 3,55\right.$ set $\left.^{\text {nd }^{-1}}\right)(\mathrm{Fs}=14,56 ; P<0,05)$.

\section{Metalotioneínas y tioles totales}

Las concentraciones de MT no presentaron diferencias significativas entre los ejemplares colectados en los distintas temporadas, ni en los poliquetos expuestos a $24^{\circ} \mathrm{C}$ y $28^{\circ} \mathrm{C}$ bajo condiciones controladas en el laboratorio $(\mathrm{Fs}=2,09 ; P>$ 0,05). En el ambiente, los promedios de MT más elevados fueron encontrados en junio y octubre (Fig. 2A). De igual manera, no se observaron diferencias entre los niveles de grupos tioles totales $(-\mathrm{SH})$ cuantificados en la pared corporal del poliqueto colectados en distintos meses ( $\mathrm{Fs}=0,58, P>0,05)$. Los promedios de -SH totales oscilaron entre 41,09 y 58,07 $\mu$ moles $\mathrm{g}^{-1}$, tanto para los organismos colectados en el ambiente como para los expuestos a distintas temperaturas $\left(24\right.$ y $\left.28^{\circ} \mathrm{C}\right)$ en el laboratorio (Fig. 2B).

\section{Peroxidación de lípIDos}

La variación de temperatura ambiental entre diciembre 2009 a octubre 2010 produjo cambios significativos en los niveles de lípidos peroxidados $(\mathrm{Fs}=11,14, P<0,001)$. Se muestran los incrementos en los niveles de TBARS en octubre, en un ascenso concomitante al aumento en la temperatura del mar. Incrementos similares fueron encontrados en los organismos aclimatados a $28^{\circ} \mathrm{C}$, en contraste a los organismos aclimatados a $24^{\circ} \mathrm{C}$ (Fig. 2C). 
A

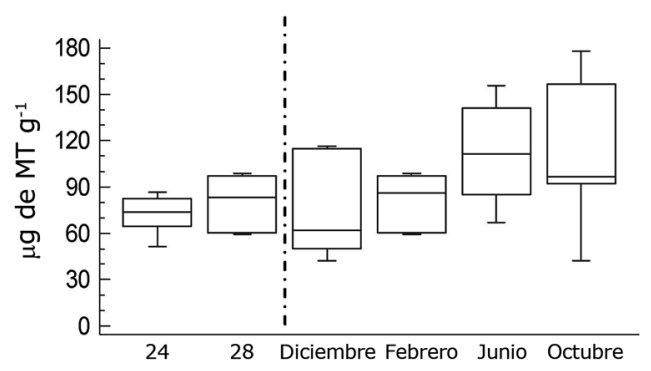

C

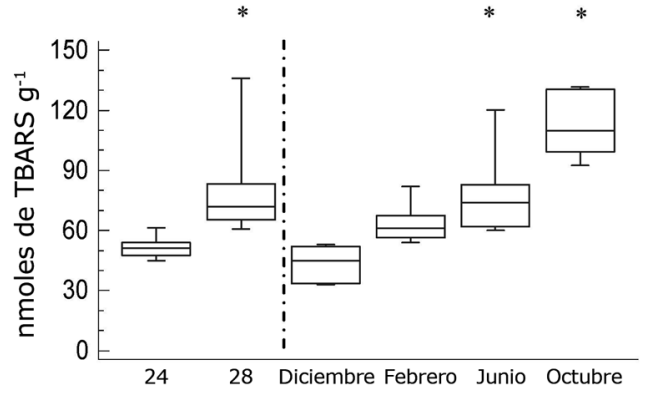

E

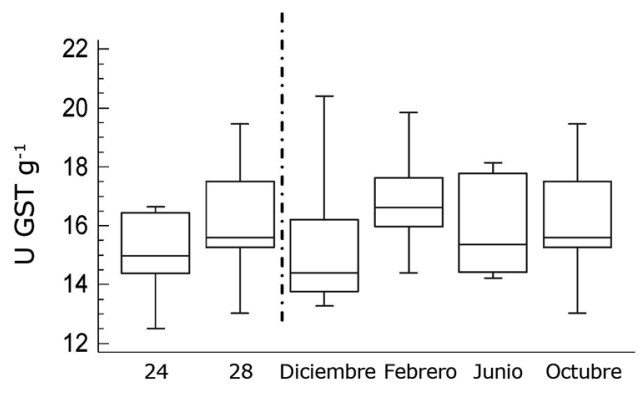

B

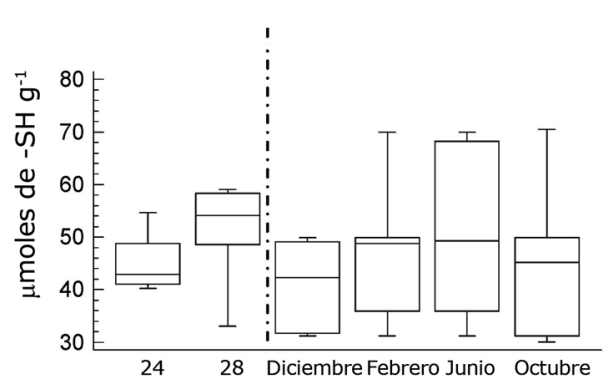

D

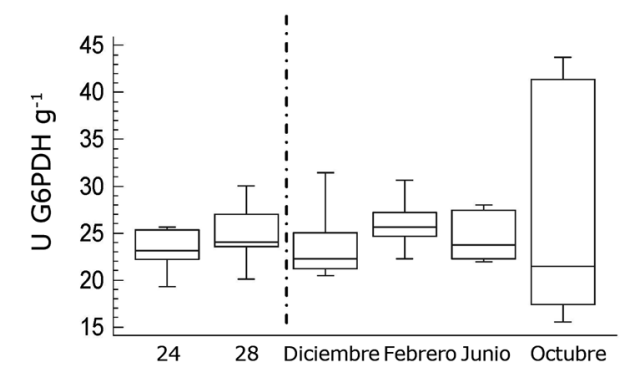

F

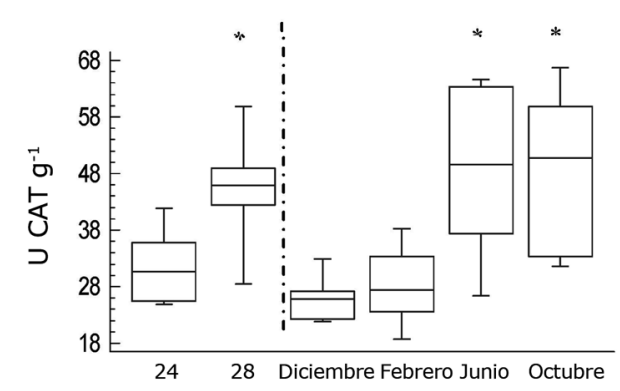

Figura 2. A) Niveles de metalotioneínas, B) Niveles de tioles totales, C) Niveles de TBARS, D) Actividad de glucosa-6- fosfato deshidrogenasa, E) Actividad de glutatión-S-transferasa, F) Actividad de catalasa en E. complanata colectado en distintas temporadas ambientales y sometidos a bioensayo de aclimatación a 24 y $28^{\circ} \mathrm{C}$ por 36 días. (*) muestra diferencias significativas al $95 \%$ de confianza / A) Metallothionein concentrations, B) Total thiols concentrations, C) TBARS concentrations, D) Glucose-6P dehydrogenase activity, E) GST activity, F) CAT activity in E. complanata collected in different seasons and under environmental acclimatization bioassay 24 and $28^{\circ} \mathrm{C}$ for 36 days

\section{Análisis enZimáticos}

La actividad de las enzimas G6PDH y GST no mostraron variabilidad con respecto a los cambios estacionales ni bioensayos de aclimatación (Figs. 2D \& 2E, respectivamente). No obstante, la actividad de CAT incrementó significativamente durante junio y octubre 2010, al igual que los poliquetos mantenidos durante 36 días a una temperatura promedio de $28^{\circ} \mathrm{C}$. Los promedios más bajos fueron encontrados en diciembre 2010 y febrero 2011 similar a los poliquetos expuestos a $24^{\circ} \mathrm{C}$ (Fig. 1F).

\section{Biomoléculas e ÍNDICES MOLECULARES}

Las concentraciones de ADN se incrementaron en los organismos expuestos a $28^{\circ} \mathrm{C}$ concomitantemente con los individuos colectados en el ambiente en junio y octubre (Fig. 3A). Los niveles de ARN no mostraron variabilidad con respecto a los cambios de temperaturas en el ambiente ni bajo condiciones controladas ( $\mathrm{Fs}=1,97, P>0,05)$ (Fig. 3B). Los promedios de proteínas totales (PT) oscilaron entre 118,66 \pm 16,16 a $140,46 \pm 41,21 \mathrm{mg} \mathrm{de} \mathrm{PT} \mathrm{g}^{-1}$ para junio y febrero, respectivamente. A su vez, no fueron encontrados cambios significativos en los niveles de PT de organismos expuestos a temperaturas contraladas (Fig. 3C). 
A

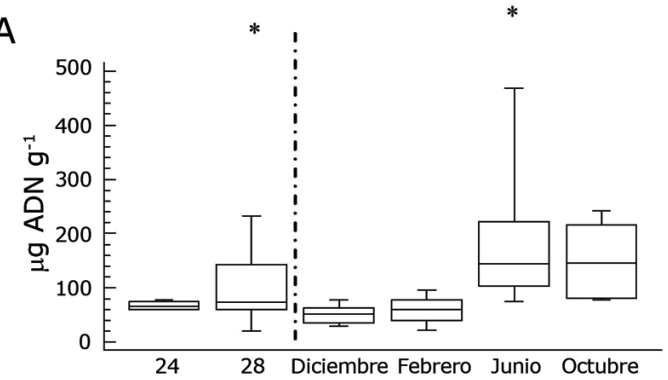

C

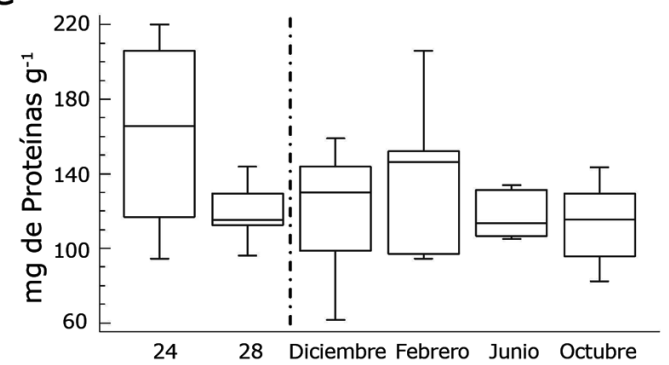

E

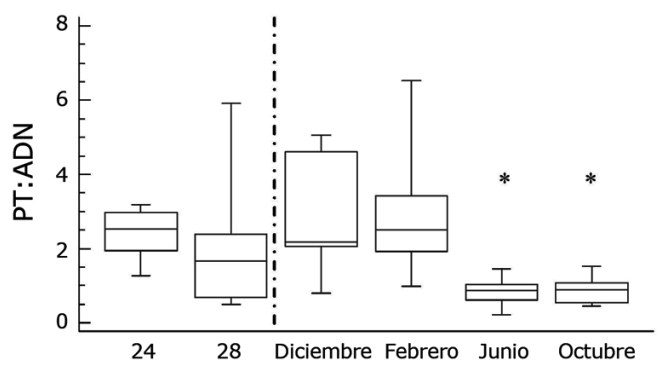

B

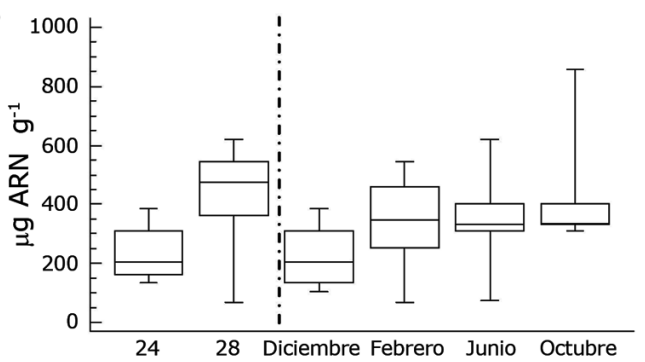

D

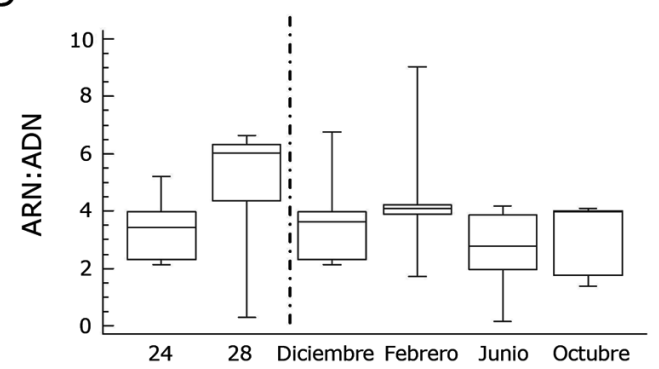

Figura 3. A) Niveles de ADN, B) Niveles de ARN, C) proteínas totales (PT), D) Coeficiente ARN:ADN y E) Coeficiente proteina:ADN en E. complanata colectado en distintas temporadas ambientales y sometidos a bioensayo de aclimatación a 24 y $28^{\circ} \mathrm{C}$ por 36 días. El asterisco (*) muestra diferencias significativas al $\mathbf{9 5 \%}$ de confianza / A) DNA concentration, B) RNA concentration, C) Total protein concentrations, D) RNA:DNA ratio, E) Protein:DNA ratio concentrations in E. complanata collected in different seasons and under environmental acclimatization bioassay 24 and $28^{\circ} \mathrm{C}$ for 36 days. The asterisk $\left({ }^{*}\right)$ shows significant differences to $95 \%$
El cociente ARN:ADN en E. complanata no presentó variaciones con respecto a los meses de colectas ni en los bioensayos de aclimatación (Fig. 3D). Los niveles de PT:ADN se mantuvieron con promedios aumentados durante diciembre y febrero, presentando un ligero descenso en julio y octubre, con respecto a todos los grupos experimentales $(\mathrm{Fs}=2,82 ; P$ $>0,05)$. En los bioensayos de aclimatación no se observaron cambios en los índices de condición (Fig. 3E).

\section{Discusión}

Los incrementos en la actividad de CAT y niveles de lipoperoxidación muestran que $E$. complanata sometido a ambientes con temperaturas mayores o iguales a $28^{\circ} \mathrm{C}$ puede desarrollar una condición de estrés oxidativo. Los cambios de la temperatura que se suscitan en el golfo de Cariaco, desde febrero $\left(23,96^{\circ} \mathrm{C}\right)$ hasta octubre $\left(29,93^{\circ} \mathrm{C}\right)$ pueden tener incidencia sobre el estatus antioxidante de $E$. complanata. En la temporada de junio a octubre, donde se registran las máximas temperaturas, se hace evidente el incremento de la actividad de CAT, haciendo notar el rol preponderante de la enzima para controlar de los niveles fisiológicos de las especies reactivas del oxígeno (ERO), especialmente peróxido de hidrógeno $\left(\mathrm{H}_{2} \mathrm{O}_{2}\right)$. La capacidad de respuesta de esta enzima en pos de los cambios térmico en el ambiente forman parte de las respuestas de ajustes fisiológicos por aumento de ERO, vinculado posiblemente con una elevada tasa metabólica y respiración celular (Abele \& Puntarulo 2004).

Al parecer, en la temporada más caliente, el poliqueto tiende a ajustar sus defensas antioxidantes en orden de mantener las concentraciones de ERO a niveles basales, sin embargo no parecen ser suficientes para contrarrestar el daño oxidativo en los lípidos (LPO). De manera similar, incrementos en las actividades de las enzimas antioxidantes y otros compuestos han sido reportados en especies de poliquetos tales comoNereis 
diversicolor (Abele-Oeschger et al. 1994), Arenicola marina (Buchner et al. 1996) y en el camarón Palaemon elegans (Madeira et al. 2016). También, se ha reportado que la actividad de CAT incrementa en las temporadas cálidas en el cangrejo Lithodes santolla (Slechvezov et al. 2015). En verano, los mejillones Mytilus edulis, M. galloprovincialis y Bathymodiolus azuricus incrementan SOD, CAT y GPx, inclusive LPO; en cambio, durante el invierno la tendencia es opuesta (Viarengo et al. 1991, Wiston \& Di Güilio 1991, Company et al. 2006).

Los efectos estacionales sobre las respuestas antioxidantes han sido revisados en otras especies: Perna perna (WilhelmFilho et al. 2001), Modiolus modiolus (Lesser \& Kruse 2004), M. galloprovincialis (Borkoviæ et al. 2005), Laeonereis acuta (Ferreira-Cravo et al. 2007), Nacella magellanica (Malanga et al. 2007), Callinectes amnicola (Olakolu et al. 2012) y Palaemon elegans (Vinagre et al. 2014). Sin embargo, en algunas investigaciones se demuestra que las defensas antioxidantes parecieran no presentar un patrón fijo con respecto a cambios estacionales. Algunas enzimas tienden al descenso en organismos expuestos a bajas temperaturas, por ejemplo la xantina oxidasa (XO) en mejillones del océano Atlántico y la actividad de GST en el pez Zoarces viviparus en el mar Báltico (Ronisz et al. 1999). Sin embargo, en el músculo blanco del pez Mullus barbatus, las enzimas SOD y GST incrementan sus actividades en invierno, y CAT, GR y GPx en primavera (Mathieu et al. 1991, Pavloviæ et al. 2009). Existe complejidad en la interpretación de la actividad de las enzimas antioxidantes, ya que también pueden observarse niveles elevados en presencia de contaminantes (Nusetti et al. 2005, Zapata-Vívenes et al. 2005).

Por otro lado, los compuestos -SH, MT y las enzimas GST y G6PDH no mostraron variaciones frente a los cambios de temperaturas en las temporadas ambientales examinadas; al parecer, estos marcadores moleculares presentan poca variación estacional, aunque pueden ser modificados por otros factores externos o fisiológicos. En contraste en branquias de L. santolla, los niveles de GSH, tripéptido que contiene un $\mathrm{SH}$, disminuyen en relación con el incremento de la temperatura del agua y el estado reproductivo durante los meses de septiembre y diciembre. Sin embargo, en el músculo de esta especie GST decrece en septiembre e incrementa en julio asociado a una condición post-muda (Schvezov et al. 2015). Algunos detalles similares han sido reportados en el mejillón marrón P. perna (Wilhelm-Filho 2001) y en el anfípodo Gammarus locusta (Gismodi et al. 2012). Algunas de tales respuestas bioquímicas han sidoútiles para en estudio de áreas impactadas por xenobióticos y su variación temporal. Se ha demostrado incrementos de MT y GST en el poliqueto Laeonereis acuta que habita en zonas contaminadas y encontrándose más elevadas en verano (Geracitano et al. 2004). A pesar que no existió cambios en las actividades de G6PDH, tal enzima puede garantizar los precursores de nucleótidos para la replicación del ADN ola síntesis de proteínas vinculadas con el crecimiento somático de E. complanata.

Los niveles de ADN indican una mayor proliferación celular en poliquetos colectados en la temporada de temperaturas más elevadas (no surgencia) y en los organismos aclimatados a $28^{\circ} \mathrm{C}$, coincidiendo con una significativa frecuencia de organismos con señales de regeneración. Al parecer, durante el período de no surgencia se estimula la autotomía en esta especie, registrándose cerca del $30-41 \%$ de ejemplares fragmentados. Estos resultados muestran una posible relación entre la temperatura y el ciclo de reproducción asexual en E. complanata; aspectos biológicos que ameritan un sistema de defensas antioxidante eficiente. Los procesos de auto-fragmentación y regeneración en poliquetos pueden ser gatillados por la temperatura (Pires et al. 2015); detalles similares han sido reportados para $E$. complanata colectados en el golfo de México (Kudenov 1974).

Los promedios de biomoléculas tales como ARN y proteínas (PT), e índice ARN:ADN, a pesar que no presentaron variaciones con respecto a los cambios de temperaturas anuales, indican que E. complanata presenta un crecimiento continuo durante todo el año. Adicionalmente, el índice PT:ADN deja mostrar un aumento en la división celular durante los meses de estratificación y un incremento de la síntesis de proteínas en las temporadas de descenso de temperatura (surgencia). La temperatura es un factor determinante sobre la actividad metabólica y las concentraciones de biomoléculas implicadas en el crecimiento (PT, ARN y ADN) y sus cocientes relacionados. Sin embargo, los niveles de proteínas en $E$. complanata presentaron sus mayores promedios en los organismos expuestos a baja temperatura. Segnini \& Chung (1991), Chung (1997), Lemus et al. 1993 y Lemus \& Chung (2006) reportaron incremento en los niveles ARN en juveniles de Oreochromis mossambicus y Petenia kraussii aclimatados a $22^{\circ} \mathrm{C}$ con respecto a los organismos sometidos a $30^{\circ} \mathrm{C}$, mostrando una relación positiva entre los valores de $\mathrm{ARN}$ y crecimiento corporal.

Los ascensos de la temperatura del agua en los períodos de no surgencia pueden estimular la autotomía y regeneración tisular en E. complanata. Al parecer, los procesos fisiológicos donde se vincula una elevada tasa metabólica existe la posibilidad de conllevar a una condición de estrés oxidativo (Schvezov et al. 2015). En tal sentido, es posible que el sistema de defensa de antioxidante en E. complanata ajuste su capacidad de removedor de ERO influenciados directamente por las variaciones de temperatura; asociado también a los procesos 
reproductivos asexuales (autotomía). El incremento en la lipoperoxidación (LPO), paralelamente a la actividad de CAT en E. complanata, sugiere ajustes metabólicos contra la acción de ERO, demandando respuestas compensatorias antioxidantes para proteger la viabilidad funcional del tejido y los procesos fisiológicos relacionados con el crecimiento. En síntesis, las temperaturas que se registran en las aguas superficiales del golfo de Cariaco inducen cambios significativos en la actividad de CAT y daños oxidativos, que coinciden con el incremento de las tasas de crecimiento corporal en los poliquetos. Al parecer, el estatus basal antioxidante E. complanata puede variar en relación a cambios térmicos ambientales.

\section{Agradecimientos}

Los autores agradecen al Consejo de Investigación de la Universidad de Oriente, a la Academia de Ciencias Físicas, Matemáticas y Naturales, y al Instituto Oceanográfico de Venezuela. Se aplicaron las normas internacionales, nacionales, y/o institucionales para el cuidado y uso de animales de experimentación. Esta investigación se adhiere a las pautas de ASAB/ABS (2012) para el uso de animales en la investigación.

\section{LITERATURA CITADA}

Abele-Oeschger D, R Oeschger \& H Theede. 1994. Biochemical adaptations of Nereis diversicolor (Polychaeta) to temporarily increased hydrogen peroxide levels in intertidal sandflats. Marine Ecology Progress Series 106: 101-110.

Abele D \& S Puntarulo. 2004. Formation of reactive species and induction antioxidant defense systems in polar and temperature marine invertebrates and fish. Comparative Biochemistry and Physiology A 138: 405-415.

ASAB/ABS. 2012. Guidelines for the treatment of animals in behavioral research and teaching. Association for the study of animal behaviour and animal behavior society. Animal Behavior 83: 301-309

Borkoviæ S, J Šaponjiæ, S Pavloviæ, D Blagojeviæ, S Miloševiæ, $T$ Kovaèeviæ, $R$ Radojièiæ, $M$ Spasiæ, $R$ Žikiæ \& Z Saièiæ. 2005. The activity of antioxidant defense enzymes in mussels (Mytilus galloprovincialis) from the Adriatic Sea. Comparative Biochemistry and Physiology C 141: 366-374.

Buchner T, D Abele-Oeschger \& H Theede. 1996. Aspects of antioxidant status in the polychaete Arenicola marina: tissue and subcellular distribution and reaction to environmental hydrogen peroxide and elevated temperatures. Marine Ecology Progress Series 143: 141-150.

Buckeley LJ \& FJ Bullow. 1987. Techniques for estimation of RNA, DNA, and protein in fish. In: Summerfelt RC \& GE Hall (eds). Age and growth in fish, pp. 345-354. Iowa State University Press, Ames.
Bulow F. 1987. RNA-DNA ratios as indicator of growth in fish. In: Summerfelt JR \& Cobb CS (eds). Age and growth in fish, pp. 255-367. Iowa State University Press, Ames.

Burton K.1956. A study of the conditions and mechanisms of the diphenilamine reaction for the colorimetric estimation of deoxyribonucleic acid. Biochemistry Journal 62: 315-323.

Buttemer WA, D Abele \& D Costantini. 2010. The ecology of antioxidants \& oxidative stress in animals from bivalves to birds: oxidative stress and longevity. Functional Ecology 24:971-983.

Cambell PN \& JR Sargent. 1967. Estimation of ARN by orcinol method of mejbum (1939). In: Cambell PN \& JR Sargen (eds).Techniques in protein biosynthesis 1: 301-302. Academic Press, London.

Company R, A Serafima, R Cosson, A Fiala-Me'dioni, D Dixond \& MJ Bebianno. 2006. Temporal variation in the antioxidant defense system and lipid peroxidation in the gills and mantle of hydrothermal vent mussel Bathymodiolus azoricus. Deep-Sea Research I 53: 1101-1116.

Chung KS. 1997. Respuestas fisiológicas de peces tropicales a cambios de temperatura. Saber 9(1): 36-39.

Ellman GL. 1958. A colorimetric method for determining low concentrations of mercaptans. Archives of Biochemistry and Biophysics 74: 443-450.

Ferreira-Cravo C, M Piedras, FM Moraes, JLR Ferreira, DPS De Freitas, MD Machado, MA Geracitano \& JM Monserrat. 2007. Antioxidant responses and reactive oxygen species generation in different body regions of the stuarine polychaeta Laeonereis acuta (Nereididae). Chemosphere 66: 1367-1374.

Freire CA, AF Welker, JM Storey, KB Storey \& M Hermes-Lima. 2011. Oxidative stress in estuarine and intertidal environments (temperate and tropical). In: Abele D, JP Vazquez- Medina \& T Zenteno-Savin (eds). Oxidative stress in aquatic ecosystems, pp. 41-57. Wiley Blackwell, Chichester.

Geracitano L, JM Monserrat \& A Bianchini. 2004. Oxidative stress in Laeonereis acuta (Polychaeta, Nereididae): environmental and seasonal eects. Marine Environmental Research 58: 625-630.

Gismondi E, JN Beisel \& C Cossu-Leguille. 2012. Influence of gender and season on reduced glutathione concentration and energy reserves of Gammarus roeseli. Environmental Research 118: 47-52.

Guderley H \& J St-Pierre. 2002. Going with the flow or life in the fast lane: contrasting mitochondrial responses to thermal change. Journal of Experimental Biology 205: 2237-2249.

Hauschild K \& MK Grieshaber. 1997. Oxygen consumption and sulfide detoxification in the lugworm Arenicola marina (L.) at different ambient oxygen partial pressures and sulfide concentrations. Comparative Biochemistry and Physiology B 167: 378-388. 
Hermes-Lima M. 2004. Oxygen in biology and biochemistry: role of free radicals. In: Storey KB (ed). Functional metabolism: regulation and adaptation, pp. 319-368. John Wiley \& Sons, New York.

Keller M, AM Sommer, HO Pörtner \& D Abele. 2004. Seasonality of energetic functioning and production of reactive oxygen species by lugworm (Arenicola marina) mitochondria exposed to acute temperature changes. Journal of Experimental Biology 207: 2529-2538.

Kudenov JD. 1974. The reproductive biology of Eurythoe complanata (Pallas, 1766) (Polychaete: Amphinomidae). PhD Dissertation, Arizona University, 128 pp. <http:// arizona.openrepository.com/arizona/bitstream/10150/288256/ 1/azu_td_7423371_sip1_m.pdf>

Lemus M \& K Chung. 2006. Effect of copper on the RNA/ DNA ratio at different temperatures in juveniles of Petenia kraussii (Pisces: Ciclidae). Boletín del Instituto Oceanográfico de Venezuela 45(1): 9-15.

Lemus MJ, KS Chung \& GJ Holt. 1993. Efecto de la temperatura sobre el crecimiento de juveniles de Petenia kraussii (Pisces:Cichlidae): Relación ARN/ADN. Revista de Biología Tropical 41(Supl. 1): 45-48.

Lesser MP \& VA Kruse. 2004. Seasonal temperature compensation in the horse mussel, Modiolus modiolus: Metabolic enzymes, oxidative stress and heat shock proteins. Comparative Biochemistry and Physiology A 137: 495-504.

Linero-Arana A \& O Diaz. 2010. Amphinomidae y Euphrosinidae (Annelida: Polychaeta) de la costa nororiental de Venezuela. Latin American Journal of Aquatic Research 38(1): 107-120.

Lowry OH, NJ Rosebrough, AL Farr \& RJ Randal. 1951. Protein measurement with folin phenol reagent. The Journal of Biological Chemistry 193: 265-275.

Madeira D, V Mendonça, C Vinagre \& MS Diniz. 2016. Is the stress response affected by season? Clues from an in situ study with a key intertidal shrimp. Marine Biology 163(41): 1-12.

Malanga G, MS Estévez, J Calvo, D Abele \& S Puntarulo. 2007. The effect of seasonality on oxidative metabolism in Nacella (Patinigera) magellanica. Comparative Biochemistry and Physiology A 146: 551-558.

Mathieu A, P Lemaire, S Carriere, P Drai, J Giudicelli \& M Lafaurie. 1991. Seasonal and sex linked variations in hepatic and extrahepatic biotransformation activities in striped mullet (Mullus barbatus). Ecotoxicology and Environmental Safety 22: 45-57.

Méndez N \& F Paéz-Osuna. 1998. Trace metals in two populations of the fireworm Eurythoe complanata from Mazatlan bay: effect of body size on concentrations. Environmental Pollution 102: 279-285.

Mendoza AR, GC Aguilera \& ML Carreón. 2002. Ácidos nucleícos para evaluar las condiciones de larvas de peces. Ciencia UANL 5(2): 210-217.
Muller-Karger FE, CR McClain, TR Fisher, WE Esaias \& R Varela. 1989. Pigment distribution in the Caribbean Sea: observations from space. Progress in Oceanography 23:23-69.

Nusetti O \& D Morales. 1988. Crecimiento en algunos tejidos del mejillón Perna perna (L.1758): composición de ADN, relaciones ARN/ADN y reservas energéticas. Acta Científica Venezolana 39: 289-293.

Nusetti O, R Salazar-Lugo, J Rodríguez-Grau \& J Vilas. 1998. Immune and biochemical responses of the polychaete Eurythoe complanata exposed to sublethal concentrations of copper. Comparative Biochemistry and Physiology C 119: 177-183.

Nusetti O, M Escalpés, G Salazar, S Nusetti \& S Pulido. 2001. Biomarkers of oxidative stress in the polychaete Eurythoe complanata (Amphinomidae) under short term copper exposure. Bulletin of Environmental Contamination and Toxicology 66: 576-581.

Nusetti O, E Zapata-Vívenes, M Esclapés \& M Rojas. 2005. Antioxidant enzymes and tissue regeneration in Eurythoe complanata (Polychaeta: Amphinomidae) exposed to used vehicle crankcase oil. Archives of Environmental Contamination and Toxicology 48: 1-6.

Oakley A. 2011. Glutathione transferases: a structural perspective. Drug Metabolism Review 43(2): 138-151.

Ohkawa H, N Ohishi \& K Yaki. 1979. Assays for lipid peroxides in animal tissue by thiobarbituric acid reaction. Analytical Biochemistry 95: 331-358.

Olakolu FC, AA Hassan \& SK Akindele. 2012. Seasonal variation of oxidative stress biomarkers in gills and muscle of blue crabs Callinectes amnicola from Lagos lagoon. European Journal of Scientific Research 80(3): 406-415.

Pavloviæ SZ, SS Borkoviæ-Mitiæ, TB Radovanoviæ, BR Perendija, SG Despotoviæ, J Gavriæ \& Z Saièiæ. 2009. Activity of oxidative stress biomarkers in the white muscle of red mullet (Mullus barbatus L.) from the Adriatic sea. Archives of Biology Sciences 61(4): 693-701.

Penchaszadeh P, C León, H Álvarez, D Bone, P Castellano, M Castillo, T Díaz, M García-Guardilla, M Lemus, F Losada, A Martín, P Miloslavich, C Paredes, D Pérez, M Sebastiani, D Stecconi, V Roa \& A Villamizar. 2000. Venezuela. In: Sheppard CR (eds). Seas at the millennium; An environmental evaluation 1: 643-659. Pergamon, Oxford.

Pires A, E Figueira, A Moreira, A Soares \& R Freitas. 2015. The effects of water acidification, temperature and salinity on the regenerative capacity of the polychaete Diopatra neapolitana. Marine Environmental Research 106: 30-41.

Ronisz D, DGJ Larsson \& L Forlin. 1999. Seasonal variations in the activities of selected hepatic biotransformation and antioxidant enzymes in eelpout (Zoarces viviparus). Comparative Biochemistry and Physiology C 124: 271-279. 
Rueda-Roa DT \& FE Muller-Karger. 2013. The southern Caribbean upwelling system: Sea surface temperature, wind forcing and chlorophyll concentration patterns. Deep-Sea Research I78: 102-114.

Schvezov N, GA Lovrich, O Florentín \& MC Romero. 2015. Baseline defense system of commercial male king crab Lithodes santolla from the Beagle Channel Comparative Biochemistry and Physiology A 181: 18-26.

Segnini MI \& KS Chung. 1991. Respuestas fisiológicas de Petenia kraussii (Pisces: Cichlidae) sometidos a cambios de temperatura. Boletín del Instituto Oceanográfico de Venezuela 30: 5-15.

Singh S, A Anand \& PK Srivastava. 2012. Regulation and properties of glucose-6-phosphate dehydrogenase: A review. International Journal of Plant Physiology and Biochemistry 4(1): 1-19.

Sokal R \& J Rohlf. 2012. Biometry: the principles and practice of statistics in biological research, $937 \mathrm{pp}$. WH Freeman and Company, San Francisco.

Vásquez-Núñez R, N Méndez \& C Green-Ruiz. 2007. Bioaccumulation and elimination of $\mathrm{Hg}$ in the fireworm Eurythoe complanata (Annelida: Polychaeta) from Mazatlan, México. Archives of Environmental Contamination and Toxicology 52(4): 541-548.

Viarengo A, L Canesi, M Pertica \& DR Livingstone. 1991. Seasonal variation in the antioxidant defense system and lipid peroxidation of the digestive gland of mussels. Comparative Biochemistry and Physiology C 100: 187-190.

Viarengo A, E Ponzano, F Dondero \& R Fabbri. 1997. A simple spectrophotometric method for metallothionein evaluation in marine organisms: an application to Mediterranean and Antarctic mollusks. Marine Environmental Research 44(1): 69-84.
Vinagre C, D Madeira, V. Mendonça, M Dias, J Roma \& MS Diniz. 2014. Effect of temperature in multiple biomarkers of oxidative stress in coastal shrimp. Journal of Thermal Biology 41: 38-42.

Wilhelm-Filho D, T Tribes, C Gaspari, FD Claudio, MA Torres \& ARM Magalhaes. 2001. Seasonal changes in antioxidant defenses of the digestive gland of the brown mussel (Perna perna). Aquaculture 203: 149-158.

Wiston GW \& RT Di Giulio. 1991. Proxidant and antioxidant mechanisms in aquatic organisms. Aquatic Toxicology 19: 137-161.

Yáñez-Rivera B \& N Méndez. 2014. Regeneration in the stinging fireworm Eurythoe (Annelida): Lipid and triglyceride evaluation. Journal of Experimental Marine Biology and Ecology 459: 137-143.

Zapata-Vívenes E. 2001. Regeneración tisular y estrés oxidativo en el poliqueto Eurythoe complanata (Polychatea: Amphinomidae) expuestos a concentraciones subletales de una fracción de lubricantes usados de motores de vehículo. Tesis de Licenciatura en Biología, Universidad de Oriente, Cumaná.

Zapata-Vívenes E, O Nusetti, L Marcano, M Escalpés \& L Arredondo. 2005. Respuestas inmunológicas y cicatrización en el poliqueto Eurythoe complanata (Annelida: Amphinomidae) expuesto a cobre. Ciencias Marinas 31(1): $1-9$.

Zapata-Vívenes E, G Sánchez \& L Marcano. 2015. Reservas energéticas e índices moleculares de condición en Lima (ctenoides) scabra (Born, 1778) expuesta a lubricantes usados de motores de automóviles. Zootecnia Tropical 6(4): $1-10$. 